\title{
La storiografia del P. De Rogatis, cornucopia di miti ispanici e motivi teatrali nell'Italia del Seicento
}

L'historiographie du père De Rogatis, corne d'abondance de mythes et de motifs théâtraux hispaniques dans l'Italie du XVII siècle

The Historiography of Father De Rogatis, a Cornucopia of Hispanic Myths and Theatrical Motifs in 17th Century Italy

Elena E. Marcello

\section{OpenEdition}

\section{Journals}

Edizione digitale

URL: http://journals.openedition.org/cei/7608

DOI: $10.4000 /$ cei. 7608

ISSN: 2260-779X

Editore

UGA Éditions/Université Grenoble Alpes

Edizione cartacea

ISBN: 978-2-37747-225-3

ISSN: 1770-9571

Notizia bibliografica digitale

Elena E. Marcello, «La storiografia del P. De Rogatis, cornucopia di miti ispanici e motivi teatrali nell'Italia del Seicento», Cahiers d'études italiennes [Online], 31 | 2020, online dal 06 octobre 2020, consultato il 28 mars 2021. URL: http://journals.openedition.org/cei/7608 ; DOI: https://doi.org/ $10.4000 /$ cei. 7608

Questo documento è stato generato automaticamente il 28 mars 2021

(c) ELLUG 


\section{La storiografia del P. De Rogatis, cornucopia di miti ispanici e motivi teatrali nell'Italia del Seicento}

L'historiographie du père De Rogatis, corne d'abondance de mythes et de motifs théâtraux hispaniques dans l'Italie du XVII siècle

The Historiography of Father De Rogatis, a Cornucopia of Hispanic Myths and Theatrical Motifs in 17th Century Italy

Elena E. Marcello

\section{NOTE DELL'AUTORE}

Il saggio si inquadra nel progetto PRIN Il teatro spagnolo (1570-1700) e l'Europa: studio, edizione di testi e nuovi strumenti digitali, prot. 201582MPMN, del MIUR, diretto da Fausta Antonucci. Alla collega, con cui condivido linee di ricerca e curiosità scientifica (il tema era negli interessi di entrambe), anche un sentito ringraziamento per la generosità accademica e per l'attenta lettura. I risultati si inquadrano inoltre nel progetto del Ministero spagnolo Archivo del teatro pregoldoniano III: Biblioteca pregoldoniana, bases de datos y archivo musical (PGC2018-097031-B-I00), diretto da Javier Gutiérrez Carou.

\section{Introduzione}

1 Il padre Bartolomeo De Rogatis (1596-1656), gesuita di Castellammare di Stabia, è noto come autore degli Elegiarum libri III (Roma, ex typographiae haeredum Francisci Corbelletti, 1641) e del più celebre Il regno de' goti nella Spagna abbattuto e risorto, ovvero La perdita e racquisto della Spagna occupata da' Mori, che viene stampato a Napoli (a partire dal 1648), a Venezia (dal 1655) e Bologna (dal 1675) in vari volumi, fino a un massimo di 7. La paternità dell'opera è sempre attribuita al padre gesuita, il quale, però, 
muore durante la peste del 1656, per cui viene incaricato il padre Andrea Sannelli di completare gli ultimi due volumi ${ }^{1}$.

2 Lo stato dell'arte sulla bibliografia materiale de Il regno de' goti non è ancora chiaramente sistematizzato. Dai dati finora estratti dai cataloghi, è la città partenopea, vicina alla sede del gesuita, il luogo di stampa della princeps, mentre l'esecutore materiale è il tipografo Francesco Savio, la cui attività è documentata dal 1631 al 1656, ma che dal 1643 è pure stampatore della Curia Arcivescovile ${ }^{2}$. Il primo volume ${ }^{3}$, in $4^{\circ}$, consta di due parti (1 $\left.1^{\mathrm{a}}, \mathrm{pp} .1-248 ; 2^{\mathrm{a}}, \mathrm{pp} .249-450\right)$, ciascuna delle quali è suddivisa in quattro libri. Capeggia il frontespizio il doppio titolo Il regno de' Goti nella Spagna abbattuto e risorto, overo La perdita e racquisto della Spagna occupata da' Mori, che allude, da un lato, alla sconfitta dello storico Roderico o Don Rodrigo, l'ultimo re dei visigoti (710-711), che perdette il regno nella celebre battaglia sul fiume Guadalete e fu causa dell'entrata dei mori nel territorio iberico; dall'altro, all'epica della Reconquista cristiana. Nelle successive seicentine, ormai in versione "tascabile", l'intestazione dell'opera subirà leggere modifiche con l'inversione dei due titoli (segnale di una diversa gerarchia della focalizzazione dei contenuti), ora preceduti dalla significativa e classificatoria indicazione "Historia de", poi ridotti ad uno: Historia della perdita $e$ racquisto della Spagna occupata dai mori.

All'incrocio tra la cultura italiana e le tradizioni letterarie della penisola iberica, il sondaggio qui effettuato sull'opera di De Rogatis si propone un duplice obiettivo: da un lato saggia la personale rielaborazione del gesuita italiano rispetto alla fonte ispanoaraba, cioè, l'opera del medico e storico morisco Miguel De Luna (ca. 1545-1615), avvisando fin d'ora che la disamina delle altre fonti dichiarate è ancora da studiare, dall'altro lato presenta una prima ricognizione dei libretti d'opera italiani, che rinviano al tema di Rodrigo, ultimo re dei Goti.

\section{Da Miguel De Luna a De Rogatis: intenzionalità autoriale, genere e tematica}

4 Il paratesto d'apertura de Il regno de' Goti... offre spunti, pur all'interno del genere discorsivo del "prologo" o "premessa", sulle intenzioni storiografiche di De Rogatis: il voler divulgare in Italia una storia che giunge «dalla Spagna, o per meglio dir dall'Arabia», definita vecchia e, allo stesso tempo, nuova perché rimasta «nelle tenebre della straniera favella». Questa volontà di trasmissione si sposa con un'intenzione filologica, il cui oggetto di studio è La verdadera historia del rey D. Rodrigo scritta da Miguel De Luna, autore nascosto dal suo ruolo di traduttore dell'arabo Albucàcim Tarif Abentarique. Com'è noto, si tratta, in realtà, di una finzione autoriale che fa da cornice narrativa alla storia stessa. Come dettagliato da $\mathrm{Maggi}^{4}$, la pseudo-traduzione di Miguel De Luna, pubblicata a Granada nel 1592 (Prima parte) e 1600 (Seconda parte), viene scritta negli anni più conflittuali, complessi, della convivenza tra cristiani e moriscos che sfoceranno nella famosa espulsione di questi ultimi nel $1609^{5}$.

5 La mistificazione del medico morisco granadino presenta una storia dell'entrata dei mori in Spagna causata dalle scelleratezze del re goto Rodrigo e dell'avvio della Reconquista ad opera del re delle Asturie Don Fruela che permette una doppia lettura: idealizzando le virtù cavalleresche, condivise sia da cristiani sia dai mori, presenta positivamente i secondi senza intaccare il piano confessionale dei rapporti tra i due 
gruppi sociali. Una lettura accettabile da entrambi le parti, come dimostra sia il superamento della censura in Spagna sia la grande diffusione editoriale in lingua originale ed in traduzione della Verdadera historia ${ }^{6}$.

6 L'intenzionalità storicistica di De Rogatis congiunta ad una certa acribia filologica, l'impegno volto a discriminare la verità dalle inesattezze, gli errori dalle falsità, si combinano, ovviamente, con il dilettevole, l'intrattenimento dei lettori, e con l'utile, il fine educativo, centrato sulla trasmissione del sapere e sulla volontà «di rintracciare nell'historia la necessità del ben fare»?

De Rogatis conclude la premessa con un'ultima avvertenza al lettore, che qui si trascrive integralmente:

Né ti dia noia il veder che l'Arabo, ch'io spesso seguo, non sia seguitato dagli altri [autori], perché gli altri non l'han veduto, come quello che dopo i loro scritti è stato nella spagnuola favella tradotto. È vero ch'egli non dice il tutto, sì perché un huomo solo non può sapere ogni cosa; sì perché il suo disegno non è di tessere historia delle cose nostre, ma delle sue; come ancora perché Saracino e nemico giurato di nostra Santa Fede, lascia a bello studio molti successi che la di lei eccellenza dichiarano, i quali io ho supplito da altri autori più moderni ${ }^{8}$.

8 L'opera si innesta in quella forma discorsiva, cara ai gesuiti, che sotto l'egida dell'intenzionalità storiografica plasma una letteratura cristiana, edificante ma altresì dilettevole. Si consideri che il Seicento privilegerà il titolo in cui campeggia la parola Historia, riconducendo l'opera a quel sottogenere che associava la funzione erudita a quella sociale ed esemplare della storia e che presentava caratteristiche tipografiche precise: era suddiviso in libri o capitoli, presentava paratesti introduttivi, note a margine e, per concludere, era corredato da un indice di nomi o cose notevoli ${ }^{9}$. In quest'ottica, è d'estremo interesse studiare tanto le intenzioni autoriali quanto la ricezione che il lettore coevo ne ha tratto.

9 Nella storia della Spagna gotico-araba la voce di De Rogatis affiora sia nell'esposizione stessa - dalla difesa delle fonti alla scelta degli episodi e all'andamento solo apparentemente lineare (ricco di anticipazioni e digressioni) - sia nella costruzione dei discorsi dei protagonisti. Dati i limiti del presente articolo ci si soffermerà su aspetti contenuti nella prima parte de Il regno de' Goti..., in considerazione del fatto che, assieme alla seconda, è quella che ha avuto maggior proiezione editoriale, oltre ad essere stata pubblicata presumibilmente sotto il controllo dell'autore.

10 La tematica di questa prima parte, come lo annuncia l'autore stesso, gira intorno a due figure maschili principali e regali: il già menzionato re Rodrigo e Pelagio (Pelayo). Rodrigo, ultimo re goto, è un caso esemplare e figura centrale della narrazione, di cui si possono più rapidamente individuare le divergenze rispetto alla letteratura ispana che lo vincola principalmente alla violenza per stupro perpetrata a danno di La Cava, altrimenti detta Florinda (nome, quest'ultimo, che appare per la prima volta in Miguel De Luna e viene riportato da De Rogatis), figlia del conte Don Julián, suo vassallo. La passione amorosa per Florinda lo porta a disattendere il regno e, così facendo, a perderlo. In De Rogatis la figura di Rodrigo acquista spessore e l'intera vicenda si arricchisce di dettagli che ne sottilineano la bontà iniziale cui fa seguito l'atteggiamento calcolatore, la progressiva perdita di valori morali cristiani e politici fino alla capitolazione finale. La costruzione del personaggio storico, pur essendo filtrata dall'apprezzamento per l'opera di Miguel De Luna, è rilevante ai fini dello studio e percepibile attraverso la disamina delle strategie narrative adottate che non sono sempre ascrivibili alla fonte spagnola. Pur ad una sommaria lettura della Prima parte, si 
evince la ricorrenza di un meccanismo narrativo che alterna l'elemento "bellico" al "patetico", quest'ultimo presente ogniqualvolta l'autore si sofferma sulle vicende di coppie di sovrani o aristocratici, di personaggi femminili o sul destino di giovani principi.

Le strategie narrative contemplano la trascrizione di lettere o la riproduzione di monologhi pronunciati nei momenti culminanti della storia. Le narrazioni acquistano forza argomentativa grazie alle scelte lessicali, agli aggettivi qualificativi e alle massime o frequenti clausole finali. È pur vero che alcuni elementi si rintracciano anche nell'opera di Miguel De Luna, giacché i due testi condividono una lettura "cristiana" della storia. Quest'ultima, per il medico spagnolo, si combina a quella "cripto-morisca", che esalta i comportamenti onesti dei protagonisti e depreca le viltà dell'animo umano, tanto più quando si concentrano in figure regali. Si tratta di una lettura "moralizzante" spesso corredata in De Rogatis dall'esaltazione delle icone della cristianità: dalla grotta e santuario della Madonna di Covadonga, a San Giacomo il maggiore, San Michele arcangelo, ecc. Ciò che, senz'ombra di dubbio, non accoglie De Rogatis dalla Verdadera historia sono alcune forme di avvicinamento culturale della fonte, quali il glossario di termini arabi evidenziati nei margini che offrono al lettore la traduzione castigliana, la spiegazione di concetti e costumi espressi nel testo in arabo traslitterato oppure l'equivalenza cronologica del calendario arabo con quello cristiano.

Quale saggio del trattamento del racconto si analizzerà l'avvio delle vicende del re Rodrigo, che nell'opera di Miguel De Luna occupa tre pagine e nella prima edizione italiana circa dieci. L'estensione della riscrittura italiana ne impedisce una trascrizione completa, ma la segmentazione dei nuclei informativi (in grassetto tra parentesi quadre) consente di percepirne la modalità redazionale, che nella prima edizione, in $4^{\circ}$, viene scandita sia dalla numerazione dei paragrafi (qui trascritti in tondo) sia dalle note marginali.

\begin{tabular}{|c|c|}
\hline Verdadera historia... & Il regno de' Goti... \\
\hline $\begin{array}{l}\text { [1] En el año de la hixera de noventa y uno, } \\
\text { reinaba en España un rey de profesión } \\
\text { cristiano, llamado por nombre Don } \\
\text { Rodrigo, godo de nación, natural de la } \\
\text { Scitia, el cual tenía en aquel tiempo su } \\
\text { reino en paz, tranquilidad y sosiego, sin } \\
\text { guerras ni discordias, como nuestro rey y } \\
\text { señor Miramolín Abilgualit Jacobo } \\
\text { Almanzor estuvo en su tiempo. }\end{array}$ & $\begin{array}{l}\text { [1] } 6 \text { Partito da' vivi Acosta, il Re Rodrigo pose subito } \\
\text { mano al governo, e nel principio diede tal saggio della } \\
\text { sua sufficienza e valore, che parea non essersi mutato } \\
\text { dal morto Re nel vivo altro che il nome. } \\
\text { [1a Carattere positivo del Re e anticipazione del } \\
\text { cambiamento a causa dell'ambizione] }\end{array}$ \\
\hline
\end{tabular}


[2] Y como la ociosidad acarrea vicios grandes daños, este desdichado rey (que así se puede llamar) dio en ejercitar malos ejercicios, y como tenía el reinado en confianza y gobernación por un sobrino suyo, llamado D. Sancho, hijo mayor de su hermano, llamado por nombre el rey Acosta, tenía mucha pena y deseaba heredar la sucesión para tener el cetro real en propiedad y no de la manera que habemos referido.

[3] El niño D. Sancho mostraba grande esfuerzo y valor en el ánimo, en tal grado que todos los de su corte le tenían mucha afición y voluntad, por cuya causa el rey D. Rodrigo, su tío, no dejaba de recibir mucha pena y cuidado, aunque exteriormente mostraba lo contrario.
[2] Lo studio principale di quei primi mesi del suo governo fu il dimostrarsi appassionatamente geloso della nutritura di suo nipote. Lo fece allevare con tutte quelle carezze che la qualità di personaggio sì grande portava seco. Né di ciò solo contento incontrava con ogni sorte di diligenza i gusti di sua cognata, che, essendo donna d'altissimi spiriti e di coraggio più che donnesco, malamente sapea contenersi dentro i confini d'una fortuna privata; \& amando svisceratamente suo figlio con impazienza grande soffriva che altri s'ornasse le tempie di quel diadema ch'era al suo capo dovuto.

[3] Crescea tra tanto il fanciullo non meno in beltà che in dispostezza di membra \& in un corpo già tutto fiori si vedeano pian piano farsi maturi i frutti d'una maschia virilità.

Sembrava per appunto un sole che, spuntando bambino dall'acque, riempie già fatto grande tutto il mondo del suo splendore. [...] [3a Effetti della bellezza del principe sugli animi umani eccetto quelli ambiziosi] $7 \mathrm{E}$ avanzavasi in lui tanto maggiormente questo malore, quanto più s'accorgea che i Grandi del suo stato aveano quasi tutti rivolti gli occhi verso quella bella stella sorgente, bramosi di vederla quanto prima risplendere sopra il trono de' suoi maggiori. [3b Timori del Re di perdere il regno, in parte istillati dai cortigiani - 3c L'infelice condizione delle corti 3d Decisione del re di uccidere il nipote-3e La dissimulazione del re] 
[4] Y como la madre deste príncipe D. Sancho, llamada Anagilda, entendiese el mal pecho del D. Rodrigo, con recelo y miedo que tenía del daño que después de algún tiempo le sobrevino, siempre tenía consigo a su hijo en custodia y buena guarda, sin confiarlo de nadie;

[5] y para más asegurar sus daños, pidió licencia al D. Rodrigo, y con su consentimiento se fue de la ciudad de Toledo, donde solía residir, a una ciudad llamada Córdoba, que es en la provincia de Andalucía, y en ella puso su casa y asiento cual convenía a su estado.
[4] Ma che non iscopre un amor vero e sollecito? Anagilda la regina, la buona madre di Sancio che non vedea più lontano dell'amato suo bene, s'accorse subito che il cuore di Rodrigo avea cangiato faccia e che non era verso il nipote quello di un tempo. Penetrò che non era tutto nettare quello che usciva dalle sue labbra e che il dolce delle parole avea meschiato l'amaro degli inganni. [...] [4a Formule comparative della preoccupazione della reginaDecisione della regina di dissimulare per "schermir l'arte con l'arte" - $\mathbf{4 b}$ Discorso al figlio sull'imminente pericolo e sulla modalità di fuga] 8 Restò a queste parole il fanciullo come chi viaggiando di nottetempo si ritrova nell'albeggiar presso gli orli d'un precipizio, che per ogni parte minaccia caduta. [...] [4c Stupefazione del principe e descrizione della sua infermità- 4d Reazione del re alla notizia della malattia del nipote]

[5] Un giorno mentre accusava fieramente la sua disgrazia, che s'ingegnava d'avvelenarli quel dolce che ritrovava nella buona disposizione di suo nipote, colto il tempo, la Regina gli disse: Sire, l'affetto della Maestà vostra verso mio figlio è tale che chi non vi sapesse zio vi giudicherebbe padre. [...] Vostro nipote non guarrà mai sotto il cielo dove ora spira. So da' medici, e l'ho per fermo, che il suo male non è contagio, ma un affanno che nel cuor chiuso ricerca campo da svaporare. [...] [5a Discorso della Regina al Re e richiesta di partire per Cordova - 5b Pensieri del re preso alla sprovvista - 5c Commiati e partenza dei due da Toledo - 5d Descrizione della città di Cordova 5e Festeggiamenti e accoglienza degli ospiti regali 5f Pacifico soggiorno della Regina a Cordova che durerà due mesi]. 
[6] Con esta novedad el rey D. Rodrigo recibió nuevo cuidado, porque imaginaba crecer su sobrino, y que se iba llegando el y cetro real en sus manos, y de rey y señor superior hacerse inferior $\mathrm{y}$ súbdito $\mathrm{y}$ vasallo suyo, lo cual sentía a par de muerte; y como creciese tanto en su pensamiento este nuevo cuidado, determinó atosigar al pobre D. Sancho; tiempo en el cual había de dejar el mando

[6] $10 \mathrm{Ma}$ lasciamola un poco in questo dolce porto di pace, che ben presto la vedremo ingolfata nel mare de' travagli per farvi miserabile e compassionevole naufragio. Ritorniamo a Toleto, dove il Re Rodrigo vedutasi scappata dall'unghie la preda che fortemente bramava di condurre al duro passo di morte, fu per morirne d'affanno. Ben s'avvide d'aver fatto un passo da inconsiderato e d'aver dato in uno scoglio dove potea rompersi affatto la nave del suo disegno. [...] [ $\mathbf{6}^{\circ}$ Sdegno del Re - $\mathbf{6 b}$ Comparazioni - $\mathbf{6 c}$ Pensieri del re sotto forma di interrogazioni - $\mathbf{6} \mathbf{d}$ Determinazione di avvelenare il nipote].

[7] y para ejecutar este mal propósito, sin muy solemnes en su corte, y envió un mensajero de su parte a Córdoba, llamado por nombre Eliero, pidiendo muy encarecidamente a la reina Anagilda que, juntamente con el príncipe D. Sancho, su hijo, fuese a su corte a hallarse en ella ${ }^{10}$.
[7] 11 Così farneticava costui, né guari tempo v'interpose che, per porre ad effetto il suo malvagio disegno, bandì per tutta la Spagna una solennissima apparecchio e magnificenza. [7a Topos del "meschiar tra l'ambrosia il veleno" - $\mathbf{7 b}$ Lettera alla cognata 7c Consegna della lettera ad Eliero, che parte per Cordova $]^{11}$. festa da celebrarsi in Toleto con estraordinario pur nella frammentarietà della trascrizione e in linea con altre letture gesuitiche: si pensi a la Cour sainte (1624) di Nicolas Caussin, alla Historia de España (1592, in latino; 1601, in spagnolo) del padre Mariana o all'opera, varia di interessi, di Pedro de Ribadeneyra. Il re Don Rodrigo si prospetta quale exemplum vitandum, cui si contrappone una figura femminile forte - il primo di altri casi nell'opera di De Rogatis - con valenza positiva: la regina Anagilda, cognata di Rodrigo e madre di Sancio. Il racconto del padre gesuita non è esente da una voluta ricerca di accuratezza stilistica, riscontrabile sul piano retorico: presenza di metafore, anche d'uso frequente (per es., sole, fiori, primo mobile, basilisco, stella sorgente, spine, nettare, veleno), similitudini (per es., l'uomo che si ritrova sperduto nottetempo), riferimenti letterari e mitologici (per es., Seneca e Lucano, Argo, Medusa o Atteone). Ad essi fanno da pendant gli excursus descrittivi (la città di Cordova), i commenti morali traslati in domande retoriche o missive che svelano i pensieri nascosti dei personaggi, i topoi sulla pericolosità delle corti, sull'inganno o sulla dissimulazione. La voce del narratore tradisce l'intenzionalità autoriale, volta a evidenziare le conseguenze del vizio (qui, l'ambizione) e la ricompensa della virtù.

14 La varietà degli inserti narrativi e non narrativi, dalle descrizioni alle missive, dalla riproduzione dei soliloqui ai dialoghi, amplifica sia la presentazione fisica e morale dei personaggi sia gli eventi descritti, rendendo amena la lettura del testo di De Rogatis, le cui potenzialità vennero percepite, come si vedrà, sul fronte teatrale. D'altro canto, sono sfrondati precisi riferimenti cronologici o culturali relativi al mondo ispanoarabo, ancora da analizzare con esaustività; tuttavia, queste omissioni non intaccano eccessivamente l'atmosfera esotica di quel mondo lontano nel tempo e nello spazio. C'è 
quindi da domandarsi come venisse recepita o immaginata quell'epoca ispano-gotica ed ispano-mussulmana nel contesto coevo italiano e ancora, per certi versi, spagnolo. Prima di procedere all'esame della ricezione de Il regno de' goti di De Rogatis nella cultura italiana del Seicento, è necessario sottolineare l'importanza dei deuteragonisti del re Rodrigo, in primis, la regina Anagilda, madre del legittimo erede al trono: una figura femminile forte, intenta a salvaguardare la vita del figlio Sancio e la successione. È figura cardinale, esemplare per opposizione a quella del re nelle vicende narrate, il cui nome verrà assunto quale emblema della forza e della fedeltà. Ai due prototipi "storici" e, come si vedrà, "drammatici" (il tiranno ed usurpatore, la sovrana e madre forte) si affiancano personaggi corollari, a partire dal principe Sancio, che assurgono a protagonisti nel divenire della narrazione.

\section{L'opera di De Rogatis e il melodramma italiano}

Quanto finora esposto permette di intuire le potenzialità letterarie e culturali della storia gotico-mussulmana di De Rogatis sul versante della ricezione di motivi e miti ispanici, scelti probabilmente per la loro esemplarità e drammaticità.

Saggio iniziale della ricezione italiana, anche se limitata al solo genere teatrale melodrammatico, è la disamina delle pièces legate alle vicende di Rodrigo, a partire dall'ineludibile catalogo del Sartori, confrontato con i database, altrettanto imprescindibili, Corago (dir. A. Pompilio) e Librettodopera.it (dir. A. L. Bellina) per percepire l'estensione del fenomeno ricettivo e della penetrazione che la lettura autoriale di De Rogatis provocò nell'ambito della letteratura drammatica italiana, in particolare, sui libretti d'opera ${ }^{12}$. Ne emerge un mosaico affascinante, costituito da tessere che si connettono e confondono per via di circolazione tra piazze, relazioni tra librettisti, contesti di produzione, un mosaico intricato, di cui qui si propone un abbozzo. Il regesto dei libretti, anche se non esaustivo, consente di verificare, in primo luogo, l'effettiva circolazione de Il regno de' goti..., opera considerata storica agli occhi dei contemporanei, i quali, come si vedrà, anticipano nei paratesti la divergenza drammatico-finzionale rispetto a quella che viene considerata una narrazione di fatti realmente accaduti.

In secondo luogo, l'esame de drammi per musica inventariati evidenzia nuclei argomentali fondanti, tradotti drammaticamente nei libretti, che subiscono una serie di metamorfosi a seconda del momento e del contesto spettacolare (le cui specificità, ovviamente, possono essere evinte solo da un'attenta collatio testuale e, sul versante musicale, delle partiture). Di fatto ci si soffermerà sugli argomenti, spesso "istorici", dei melodrammi che permettono di individuare alcuni nuclei narrativi e appaiono quali sommari capostipiti della diffusione melodrammatica dei protagonisti storici spagnoli. La lettura degli argomenti, inoltre, offre l'occasione per due considerazioni: la prima concerne la contrapposizione dei personaggi principali e la visione dell'alterità; la seconda riguarda la loro funzionalità nel contesto drammatico e, soprattutto, musicale, fattore essenziale di questa produzione teatrale.

All'origine di molti argomenti è la figura di Rodrigo, ultimo re dei goti, accolta in momenti differenti della vicenda della successione a partire dai tentativi di usurpare il trono al nipote e dai rapporti che il monarca intesse con le figure femminili - la moglie Eliata, la cognata Anagilda e la celebre figlia del conte Giuliano, Florinda-, vere 
deuteragoniste (quando non protagoniste principali) dell'azione, che configurano sia l'intrigo storico-politico sia la vicenda amorosa del sovrano.

19 La vicenda di Zahara/Eliata, la giovane principessa mora che si convertirà al cristianesimo e sposerà Rodrigo, si snoda nella prima parte dell'opera di De Rogatis. Sorvolando sul brevissimo accenno nel terzo libro (p. 133), Eliata è protagonista del secondo (pp.78-91), in cui si racconta l'innamoramento del re, la conversione della fanciulla ed il matrimonio fino all'arrivo di Florinda. Ricompare poi nel quarto libro (pp. 185-206), ove, dopo il saccheggio di Cordova, Eliata, ormai regina vedova di Rodrigo, sposa in segreto l'infante di Tunisi, convertitosi al cristianesimo, ed è infine condannata a morte col suo sposo. Tralasciando il libretto di F. de Lemene, L'Eliata $(1661)^{13}$, opera scritta durante un soggiorno romano del poeta lodigiano, gli argomenti che ritraggono la vicenda della regina vedova sono d'area napoletana e di fine secolo: il primo è L'Eliata, ovvero L'inganno fortunato di Antonio Sabbatini (Napoli, 1693); il secondo, già varcata la soglia del Settecento, è il melodramma di Ignazio Maria Mancini, Li due martiri mori (Napoli, 1720).

L'Eliata... Li due martiri mori 
Rodrigo, quel barbaro re della Spagna che procurò l'esterminio di Sancio, suo nipote, che dovea ereditare la corona, rimasto poi per quella morte assoluto Signore del regno, li vien portata dalle procelle Eliata unica figliuola di Maometto Re moro; Rodrigo, accoltala cortesemente nella sua regia, ne accende fuor di misura impudica una fiamma, e non volendo per varie stratagemme piegarla al suo volere, risolve nel fine di farla sua moglie con ridurla nella fede celeste. Intanto or si figura a caso qui presente Maometto Gilairro, infante di Tunisi, quell'infante appunto che venuto doppo con Tariffo General de' mori alla sconfitta e morte di Rodrigo, fu sposo di quell'Eliata, come nell'Istorie del P. Rogatis; e mentre questi intende il di lei fortunato tragitto e prima preso dal grido di sua bellezza, ne procura il frutto d'una correspondenza, della quale avvedutasi Urraca, sorella di Rodrigo, che alla vista di questo Infante non avea pace, s'ingegna sotto il nome di quella di farlo ingannevolmente suo sposo come sortisce ${ }^{14}$.
Roderico, ultimo re de' goti, all'ora che violentò la figliola di D.Giuliano, conte di Tangeri, che da suo legato in Africa assistea, provocò contro di sé e il suo regno l'arme de' saraceni, i quali portati alla conquista delle Spagne in una battaglia campale sconfissero l'essercito del Re, che, vistosi privo del regno, si fuggì dentro un bosco e cangiato con un pastore le vesti regali, cominciò a piangere le sue colpe, sotto la disciplina d'un solitario, ove si morì.

Entrarono per un tal trionfo vittoriosi in Cordova i saraceni a far prigioniera la regina Eliata, figliola di Macometto, già cristiana, ma dapprima ne restò talmente preso l'Infante di Tunisi confederato coll'armi del Re Giacomo Almazorre Miramamolino dell'Africa, che anzi che lui prigioniero rimase della costei bellezza, laonde, chiestala per isposa, ella contraddisse per essere lui di rito pagano. Da che, dopo molti arti usate, non potendo l'Infante soffrir gl'impeti d'una amorosa violenza (condotto anch'a ciò da lume superiore), cristiano si rese; ciò penetratosi da' capi dell'essercito saraceno e perché con ciò venivano a dichiararsi amendue rei per il sacrilegio, com'essi falsamente stimavano delle violate leggi, ne parteciporno le corone dell'Africa, dalle quali si ebbe il rescritto della decapitazione (conforme la fece seguire Albucacino, rimaso al governo di Cordova insieme coll'Infante), sendo ambi costanti a non mancare al lume communicato loro dalla cattolica fede ${ }^{15}$.

Dato che la vicenda di Eliata si intreccia con le aspirazioni politiche del re Rodrigo, ed in particolare con le conseguenze della di lui ambizione e scelleratezza, un'altra serie di argomenti si ricollega direttamente con i tentativi di usurpazione del trono contro il legittimo erede Sancio e con le conseguenti peripezie della regina Anagilda. Gli eventi ripercorrono l'intero primo volume de Il regno de' goti..., di cui si è analizzato solo l'avvio. Si anticipa inoltre che il nome della sovrana Anagilda verrà a sostituire anche quello di Doña Sancha/Sancia, legato alle vicende del Conte di Castiglia, anch'esse narrate da De Rogatis. Ivald ${ }^{16}$ ha identificato la prima versione di una serie di melodrammi connessi con i tre protagonisti, in cui si focalizza l'attenzione sull'erede o sull'usurpatore. Da Il Sancio (Modena, 1656) deriva un libretto genovese del 1671. La seconda versione delle vicissitudini di Rodrigo-Anagilda-Sancio, di diffusione più fortunata, prende, invece, avvio da Il Roderico (Milano, 1684) e, nel corso del Seicento, "rinfresca" a volte il titolo dell'opera, consueto slogan pubblicitario della rappresentazione, soffermandosi sui co-protagonisti o sull'esemplarità della vicenda. Si 
riproduce l'argomento, infatti, ne L'Anagilde, overo Il Rodrigo (Reggio, 1685), ne L'innocenza rediviva nelle ribellioni (Fano, 1690), ne Il tiranno deluso (Vicenza, 1691). Di seguito, gli argomenti dei due libretti capostipiti: 
Acosta, della generosa e reale stirpe de' Goti, re di Spagna, ridotto all'estremo di sua vita, lasciò in custodia al fratello Rodrigo, insieme col regno, Sancio, suo figliuolo, che minor d'età restava unico erede dello scettro paterno. Nel maneggiar la tutela Rodrigo, affascinato dalla dolcezza del comando, per continuarsene il possesso, machina contro la vita del nipote, di che avvedutosi la madre di Sancio, Anagilda, risolve con improvisa fuga sottrarlo al pericolo. Si ritira in Algerzia [sic], città di D. Giuliano, Conte di Tangeri \& Prencipe di sangue goto; qui la profuga regina \& il diseredato infante vengono compassionati e accolti dal Conte e dalla Contessa e dalle due figliuole Florinda e Delmira che, giovinette di straordinaria bellezza e di brio virile, crescevano sole eredi degli stati del Conte. Scambievole amore con catene indissolubili lega le anime di Sancio \& di Delmira. Indi è che estremamente sensibile loro vien necessitata la separazione, necessitata, dico, dalle persecuzioni di Rodrigo, che minacciava l'esterminio del Conte se seguitava a protegere gl'innocenti fuggitivi. Fanno vela verso l'Africa Anagilda e Sancio. Approdati, s'incaminano alla regia del monarca Almanzorre, ma su le Africa, ne' solitudini risvegliatasi una notte Anagilda, trova mancarsi Sancio. Si dispera la desolata madre, piange e si dibatte. Alla fine atterrita dal sopracarico di tante sciagure, supponendo che suo figlio sia stato o involato da qualche arabo ladrone, overo divorato da qualche fera, risolve dimorare isconosciuta nell'Africa fino a tanto che il Cielo protettore degl'innocenti, punita alla fine la tirannia del cognato, la rimetta in condizione di migliore fortuna. Viveva egli intanto felicissimo nella Spagna e liberato del nipote, del quale mai più si seppe certezza, pensava di dare co' suoi sponsali successore al regno. Capricciosa fortuna ne porse la Fortuna portando a' lidi di Spagna Zabra, bellissima Infanta di Tunisi, che, imbarcatasi su picciol vascello per costeggiare per diporto le rive tunetane, fu da improviso temporale sbattuta a Capo di Gatta, promontorio della Spagna. Indi presentata a Rodrigo, che di lei invaghitosi, la fece sua sposa e coronolla regina chiamandola Eliata. Correvano a gara le più qualificate damigelle del regno a servire la regina e, fra l'altre, Florinda, una delle figlie del Conte D. Giuliano. Sazio fra poco Rodrigo, all'uso dei tiranni, della bella Eliata,

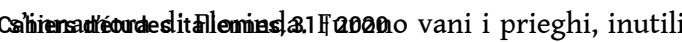
le offerte contra saggia donzella, onde il tiranneggiato tiranno si risolse ricorrere alle violenze. Si ritira presso il padre in Algezira la violata fanciulla. Racconta al padre lo scorno sofferto \&,
Morto Acosta, Re delle Spagne, fu intrapresa l'amministrazione del regno da Roderico, il fratello, come tutore di Sancio, tenero Infante, nell'antica metropoli di Toledo. La libidine del commando suggerì a quegli le massime del tradimento. Pensò di assicurarsi lo scettro in mano con togliersi dagli occhi il crescente nipote. Tentò più vuolte [sic] il veleno, ma ben guardato il fanciullo dalla madre Anagilda, sempre più si avanzava nell'affetto de' sudditi alla salita al trono. Il che mal sofferendo l'insidioso usurpatore, passò contro di entrambi all'imposture di lesa maestà e fli obligò a fugire la loro deplorabile costituzione. S'imbarcarono verso l'Africa per implorare contra il loro oppressore l'armi di Ulit, Re de' mori, ma patirono in mare mortal naufraggio. Penetrati da Roderico i loro disegni, spedì anch'egli a quella volta Don Giuliano Conte di Tangeri, Prencipe di Alghizirra, in qualità di ambasciatore, per divertirne gli effetti, ma mentre questi colà si maneggiasse per tal affare, scordatosi il tiranno e della gratitudine e del rispetto, usò violenza all'onore di Florinda, unica figlia di quel grave primato, che, avute le notizie dell'offesa, cangiò anch'egli figura e, divenuto nemico implacabile di Roderico, portò l'armi de' mori all'invasione di quella monarchia. Hist. Spagn. del Rogatis. vol. primo.

Suppositi verisimili

Che Sancio, diviso e pianto per morto nella borasca dalla madre Anagilda, che pur da lui era creduta estinta, si salvasse da quel naufraggio e ritirato ne' boschi di Toleto, ivi non conosciuto facesse vita pastorale. Che pervenuta in Africa Anagilda seco traesse su l'ale della speranza l'innamorato Ulit con numeroso essercito a' danni di Roderico. Che Zilauro, Infante di Tunesi, amante riamato di Anagilda, spedito nella reggia di Toleto ad intimare la guerra a Roderico, s'invaghisse delle bellezze di Florinda e, nel difenderla dagl'insulti del $\mathrm{Re}$, ne guadagnasse iguale corrispondenza. Che per avanti fossero passati amori fra Sancio \& Florinda ${ }^{18}$. 
21 Hapax argomentale nella storia di Rodrigo, almeno per il momento, è un particolare magico-profetico, ricordato in un libretto, di cui aveva dato notizia l'Allacci definendolo «Unico nel suo genere [...] forse non essendosi mai più veduto il secondo in cui un solo attore per tre atti continui rappresenti tutte le scene» ${ }^{19}$, sul quale il citato Ivaldi non si era soffermato ma l'aveva dettagliato in appendice. La descrizione del Palazzo incantato di Toledo e della torre, che verrà aperta da Rodrigo rivelando, non un tesoro, ma statue di bronzo e nefasti presagi, si ritrova nel terzo libro della prima parte di De Rogatis (pp.123-133). Ne Il Rodrigo. Dramma per musica d'un solo personaggio del riminese Giuseppe Malatesta Garuffi (Roma, 1677), appartenente all'accademia romana degli Infecondi, l'argomento non solo rimanda al gesuita italiano ma persino alla sua fonte spagnola:

Non molto lungi da Toledo, città delle Spagne, in seno ad un'orrida selva antico edificio trovavasi. Di chi l'avesse eretto il tempo non ne avea lasciata contezza. Solo narravasi, come per sogno, essere stato quell'Ercole che fondò Eraclea. Dalla maggior parte però asserivasi esser opra d'un mago, perciò vulgarmente chiamavasi il Palaggio incantato. Si penetrava nel di lui vuoto per sotterranea caverna lunga poco meno che un miglio. L'ingresso di essa era incavato nelle dure viscere d'uno smisurato macigno, difeso da macigna porta in ferro, in fronte alla quale intagliata una cifra leggevasi, che in nostra lingua così risuonato avrebbe:

A disvelare i beni miei fatali

Aprirannomi sol forze reali.

Molti Re goti che allora dominavan parte delle Spagne tentato aveano di penetrarvi, atteso che, appena violentate le porte, pareano disserrati gli abissi, tanto erano colà dentro orrendi i sibili e i venti. Rodrigo, Re goto, anch'esso sogetto del nostro dramma, quello che, fratello di Acosta suo antecessore, ebbe da lui morendo raccomandata la custodia del regno e la tutela di Sancio vero successore, come figlio unico \& in età ancora tenera, cangiato, per la cupidigia di regnare in odio l'amore verso l'innocente nipote, cercò ad esso \& ad Anagilda, la madre, far trangugiare insidiosamente in una tazza la morte. Ma l'oculata Reina discoperse le trame dell'empio, ritirossi col figlio fintolo infermo in Cordova, città situata nel cuore della Betica. Il tiranno per farli ritornare a Toledo bandì una solenne festa \& invitolli. Essi, fingendo di non essersi avveduti delle insidie, v'intravennero, ove pur non riuscito a Rodrigo, per cagione d'Anagilda, d'avvelenare il nipote, fu di nuovo necessitato a lasciarli partire. Ma poco dopo, fatta precorrere calunia di lesa maestà contro alla propria vita dall'Infante tramata, lo fece imprigionare in una torre non lontana da Cordova, di dove pure fu ritolto dalla genitrice alla morte, con cui poscia fuggendo e dal regno e dal carnefice, spezzatasi in uno scoglio la nave, amedue naufragarono in uno [sic] costiera dell'Africa. Invogliato indi a non molto Rodrigo nell'accennata spelonca perché fantasticava co' proprii pensieri che colà dentro un qualche gran tesoro vi si racchiudesse, accompagnato da alcuni suoi ruppe a viva forza l'entrata e con una spada alla destra \& una accesa fiaccola all'altra, penetrato quell'antro, sboccò finalmente in un'ampia sala di forma quadrata con le pareti e le volte di strane e misteriose figure intarsiate. Stava piantata in mezo di essa sovra marmorca [sic] base una statua di bronzo di vista fiera e minaccevole, stringente nella destra una pesante mazza con cui a tutto sforzo percuoteva ora da un lato ora dall'altro il suolo e le mura. Ciò che a Rodrigo colà dentro avvenisse si rappresenta nel dramma.

Si suppone che Rodrigo amoreggiasse Florisbe, figlia di un principale Barone del regno e prima dama d'Eliata sua moglie. S'avverte che tra le statue che circondano la maggiore quella che viene da Rodrigo atterrata deve rapresentare la Vendetta; l'altra, dalle cui mani cade il ferro, dev'esser del Furore.

Autori da' quali s'è levata l'istoria suddetta Michele de Luna, part. I, lib. 1, ca. 6 . Beuter, lib. 1, cap. 27. Bartol. de Rogatis, part. I, lib. 3, num. $9^{20}$. 
22 Altra figura femminile legata al re Rodrigo, le cui vicende vengono narrate nella parte seconda, libro secondo, di De Rogatis (pp.321-352), è la figlia Egilona. Il matrimonio della principessa gota con Abdalasiso è alla base, con mutamenti dell'onomastica, de L'amar per virtù del napoletano Donato Cupeda, con musica attribuita ad Antonio Draghi, che fu rappresentato il 7 novembre del 1699 al San Salvatore di Venezia. La derivazione storica da De Rogatis è dichiarata, anche se, a detta di Selfridge-Field, il prodotto finale contiene «debts of the elaboration here to popular romances from the period 1656-96» ${ }^{21}$. D'altro canto, anche l'argomento del libretto anonimo (di Giovanni Battista Borghi?) Egilina (Milano, 1793), che oltrepassa di gran lunga i limiti cronologici prefissati, riproduce gli eventi di Egilona, qui non figlia, ma moglie di Rodrigo. In quest'ultimo testo, la precisazione sulla parentela si attribuisce alle premesse di Voltaire alla tragedia Tancrède, ormai referente canonico dell'illuminismo italiano, che fa sospettare una contaminazione argomentale tra Eliata ed Egilona tutta da verificare: 
Nel tempo che i mori passarono a l'acquisto della Spagna, la regina Eliatta, moglie dell'infelice Rodrigo, ultimo Re de' goti, partorì una bambina, cui pose il nome di Egilona e, nata appena, la raccomandò a Cratilo, suo confidente di provata fedeltà perché l'allevasse segretamente ed in fortuna privata, lungi $\mathrm{da}$ ' pericoli che soprastavano alla Casa Reale. Essendo l'Infanta cresciuta non meno in età che 'n bellezza ed in tutte le virtù proprie di gran Principessa, fu ardentemente amata da un nipote di Cratilo, solo consapevole della vera qualità d'Egilona e del pensiere ch'avea il zio di darla in moglie al suo figlio maggiore. Ond'egli non potendo sofferirla in poter del rivale, mosso da rabbiosa gelosia, andò a rivelare tutto al Principe Abdalasisso, conquistatore e governatore della Spagna per Aben Ciris, re dell'Arabia, suo cognato. Essendosi perciò ordinato ad Egilona che si trasferisse alla corte, appena il principe la vide, che se ne invaghì, nè tardando le sue rare prerogative ad acquistargli la bramata corrispondenza, celebrò con essa le seconde nozze, ma con gravissimo sdegno di Madema, sua prima moglie, restata in Africa, la quale non tralasciò verun mezzo d'irritare il Re, suo fratello, contro il marito. Nè mai si vide tra' duo sposi amor più tenero di quello che durò tra Abdalasisso ed Egilona fino all'estremo della lor vita. Tanto s'ha dall'Istorie del P. De Rogatis della perdita e riacquisto della Spagna p. 2. 1.2.

Si finge

Che trattenendosi Cratilo nella reggia di Cordova con duo suoi figli, cioè Consalvo ed Elvira e con la Principessa Egilona, di cui era comunemente stimato padre, fosse molto beneficato dal Principe Abdalasisso, che si trovava fortemente invaghito d'Egilona senza però saperne la vera qualità. Che la Principessa dal suo genio, dalle persuasive di Cratilo e da' propri meriti dell'Amante venga stimolata a corrispondergli, ma che, per gratitudine verso Cratilo, si sforzi di riamare il suo figlio Consalvo, da cui era amata su la speranza di salire un giorno con le sue nozze al trono della Spagna. Che Madema, sorella d'Aben Ciris, non già sposa, ma amante di Abdalasisso, si trasferisca in Cordova per disponerlo alle sue nozze e che per viaggio le găungal'avviso della morte di suo fratello. Ch'ella per gli suoi interessi procuri di tenerla occulta, ma che già, per lettere particolari, fosse ancor nota ad Amuratte, Prencipe di Tunisi, che, da gran tempo suo amante, l'accompagnava in
È noto nella Storia di Spagna come il Conte Giuliano per vendicarsi di un oltraggio fatto alla figlia da Roderico, suo Re, eccitasse i mori contro la sua Patria medesima. È noto altresì come Musa-Abenzair, Generale de' mori, invase e conquistò il regno di Roderico, che, dopo varie sconfitte, nella famosa battaglia presso Ezija perdè il regno e la vita. Morto Roderico ed essendo rimasta in potere de' barbari Egilona (secondo la Storia, sposa di Roderico, e secondo alcuni autori, massime il signor Voltaire nelle sue Note al Tancredi, non isposa ma figlia), questa Regina per salvare il proprio onore e ricuperare la libertà accettò in isposo Abdasaziz, figlio di Musa-Abenzair suddetto, che della bellezza di lei erasi estremamente invaghito. È da notare altresì che prima dell'invasione de' mori fatta a suggerimento del Conte Giuliano, alcuni anni avanti erano stati da Roderico cacciate di Spagna diverse famiglie de' mori, che in essa abitavano, e fra gli altri anche la famiglia di Musa-Abenzair, che in quella fuga perdette un suo tenero figlio. Da questi storici fondamenti è tratto l'argomento del presente dramma e si sono ritenuti i nomi principalmente noti nella storia, se non che al nome di Egilona si volle sostituire quello di Egilina, a quello di Musa-Abenzair quello di Musa, a quello di Taric-Aben-cier quello di Tarico per adattarsi all'espressione più facile della musica. È però necessario riflettere che questi dominatori mori non erano tutti neri di colore, ma solo alcuni, essendo nel resto per la maggior parte popoli bianchi dell'Arabia e della Barbaria, come nella Storia medesima appare ${ }^{23}$. 
23 Florinda, da cui avrà origine la vendetta del conte Giuliano e l'invasione dei mori che,
curiosamente non rimanda più alla Historia del gesuita, ma ad un altro autore
emblematico della Compagnia di Gesù, il Padre Antonio Foresti, autore del diffuso
Mappamondo istorico (Venezia, 1694-1735). Inoltre, il panorama appare intricato anche
sul versante della paternità dei libretti. In posizione cronologica più alta si pone
Francesco Silvani con Il duello d'amore e di vendetta (Venezia, 1700), cui segue Vincer se
stesso è la maggior vittoria, opera composta durante il soggiorno italiano di Haendel
(Firenze, 1707), Il duello d'amore e di vendetta di Giovan Battista Gianoli (Firenze, [1718])
e, dello stesso Silvani (?), L'odio placato (Venezia, 1730).

\section{Conclusione} ricchezza di linee di ricerca e vorrebbe avviare un'analisi più sistematica ed esaustiva dell'opera completa del De Rogatis, che implicherebbe un censimento e una classificazione dei "miti" (personaggi e luoghi) o motivi storici spagnoli, individuandone le fonti usate (solo in parte dichiarate), in modo da evincere quali dettagli pseudostorici o leggendari il cronista gesuita accoglie e come vengono rielaborati, quali strategie discorsive usa, per poi definire il raggio di circolazione in Italia sia dell'opera di De Rogatis sia delle produzioni "concorrenti". Accanto alla vicinanza tematica e cronologica di alcuni drammi - si vedano gli anni '80-'90 del Seicento o di inizio Settecento - cui fa da contrappeso la diversità geografica (Siena, Roma, Venezia, Genova, ecc.), vanno considerate le relazioni tra i librettisti ${ }^{24}$, la fucina educativa gesuitica ed il contesto di origine dei drammi. In particolare per quanto riguarda quest'ultimo aspetto occorre render conto della sotterranea comunicazione tra centri così diversi e della coesione drammatica cui è approdato il melodramma che a fine secolo aveva raggiunto compiutezza di stilemi e meccanismi per cui «ora si tolleravano in scena e in attitudini canore protagonisti non solo autenticamente esistiti, ma in epoche neppure così remote come potevano essere quelle in cui si situavano molte storie spagnuole» ${ }^{25}$.

L'indagine, così impostata, è evidentemente meno semplice di quel che sembra, giacché se si scorre l'indice dei sette volumi de Il regno de' goti nella Spagna abbattuto e risorto, ovvero La perdita e racquisto della Spagna occupata da' Mori, tra le cui varie fonti spicca ora anche il padre Mariana, si dettagliano le vicende della sorella di Pelagio con il moro Munuza, dello stesso re cristiano Pelagio, della vittoria di Covadonga, del Cid, del Conde de Saldaña, di Bernardo del Carpio, del moro Muza, di Fernán González, e così via ${ }^{26}$. Anche la lettura dei soli argomenti dei libretti palesa il coacervo di miti cardinali, perfino identitari, spagnoli evocati e soggetti a metamorfosi onomastiche e drammaturgiche, cui fa seguito la rielaborazione sul piano testuale e musicale del libretto e della partitura, qui inevitabilmente tralasciata. C'è poi da verificare se sono costoro le figure maggiormente esaltate da De Rogatis e, soprattutto, se, e come, vengono accolte in modo da definire il processo di appropriazione dei miti ispanici in Italia dal Seicento in poi. Insomma, una laboriosa e appassionante indagine che ha origine da Il regno de' goti..., vera cornucopia di motivi e di visioni della Spagna e della sua storia. 


\section{BIBLIOGRAFIA}

ALLACCI Leone, Drammaturgia di Lione Allacci accresciuta e continuata fino all'anno MDCCLV, Venezia, Giambattista Pasquali, 1755.

AMBrosio Luca, Drammi, commedie e favole musicali all'ombra del Colosseo. Modi e forme dell'opera a Roma tra il 1668 e 1689, Tesi di dottorato, Pavia, Università di Pavia, 2017.

BotTALINo Giovan Battista, Il Roderico, Milano, Federico Maietta, 1685.

Chinchilla Perla (dir.), Lexicon de formas discursivas cultivadas por la Compañía de Jesús, Ciudad de México, Universidad Iberoamericana, 2018 (ebook).

CUPEDA Donato, L'amar per virtù, Vienna, Susanna Cristina vedova di Matteo Cosmerovio, 1697.

D’ANGELO Giuseppe, La chiesa del Gesù e la missione del 1649 in Castellammare di Stabia, «Cultura e Territorio», Annali del Distretto Scolastico 38, $\mathrm{n}^{\mathrm{i}}$ 15-16-17, pp. 125-177.

DE LunA Miguel, Historia verdadera del rey don Rodrigo, estudio preliminar L. F. Bernabé Pons, Granada, Universidad de Granada, 2001.

DE RogATIS Bartolomeo, Il regno de' goti nella Spagna abbattuto e risorto, ovvero La perdita e racquisto della Spagna occupata da' mori, Napoli, Francesco Savio, 1648.

DI MARCo Giampiero, Librai, editori e tipografi a Napoli nel XVII secolo (Parte I), «La Bibliofilia», vol. 112, n 1, 2010, pp. 21-61.

Di MARCo Giampiero, Librai, editori e tipografi a Napoli nel XVII secolo (Parte II), «La Bibliofilia», vol. 112, n 2, 2010, pp. 141-184.

Egilina, Milano, Giovan Battista Bianchi, 1793.

FABBRI Paolo, Il secolo cantante. Per una storia del libretto d'opera in Italia nel Seicento, Roma, Bulzoni, 2003.

FRANCHI Saverio, Drammaturgia romana, Roma, Edizioni Storia e Letteratura, 1988-1997, 2 voll.

Il Sancio, Genova, s. n., 1671.

IVALDI Armando Fabio, "Sancio", overo gli equivoci del sembiante, in Spagna e dintorni, a cura di Maria Grazia Profeti, Firenze, Alinea, 2000, pp. 265-304.

MAGGI Eugenio, Le verità del falsificatore: storia, finzione e scontro di culture in Miguel de Luna, in Oriente e Occidente nel Rinascimento, Atti del XIX Convegno internazionale (Chianciano-Pienza, 16-19 luglio 2007), Firenze, Cesati, 2009, pp. 611-623.

MALATESTA GARUfFi Giuseppe, Il Rodrigo, Dramma per musica d'un solo personaggio, Roma, Tinassi, 1677.

MANCINI Ignazio Maria, Li due martiri mori, Napoli, Monaco, 1720.

MAzzocchI Giuseppe, El teatro español en Lombardía a fines del siglo XVII, «Diálogos hispánicos de Amsterdam», $n^{\circ}$ 8, fasc. 3, 1989, pp. 691-714.

PARISI Catello, Cenno storico-descrittivo della città di Castellammare di Stabia, Firenze, s. n., 1842.

ROMERO TOBAR Leonardo, Temas literarios hispánicos, Zaragoza, Universidad, 2013-2015, 2 voll. 
SABBATINI DI SALERno Antonio, La Eliata overo L'inganno fortunato, Napoli, Giovanni Vernuccio e Nicola Layno, 1693.

SARTORI Claudio, I libretti italiani a stampa dalle origini al 1800. Catalogo analitico con 16 indici, Cuneo, Bertola e Locatelli Editore, 1990-1994, 7 voll. Consultabile all'indirizzo <www.examenapium.it/ libri/sartori.htm>.

SELFRIDGE-FIELD Eleanor, A New Chronology of Venetian Opera and Related Genres, Stanford (California), Stanford University Press, 2007.

SOMMERvogel Carlos, Bibliothèque de la Compagnie de Jésus, Bruxelles / Paris, Schepens / Picard, 1809-1932, 12 voll.

\section{Sitografia}

BELLINA Anna Laura (dir.), Libretti d'opera, <www.librettodopera.it/public/>.

Pompilio Angelo (dir.), Corago. Repertorio e archivio dei libretti del melodramma italiano dal 1600 al 1900, $<$ http://corago.unibo.it/>.

\section{NOTE}

1. C. Sommervogel, Bibliothèque de la Compagnie de Jésus, Bruxelles / Paris, Schepens / Picard, 1809-1932, 12 voll., vol. VII, p. 18. I due volumi cui si fa riferimento sono le stampe veneziane del Guerigli del 1683 e 1689. Il De Rogatis è figura impegnata a Castellammare di Stabia, le cui tracce si ritrovano anche in diversi documenti d'archivio. Si veda C. Parisi, Cenno storico-descrittivo della città di Castellammare di Stabia, Firenze, s. n., 1842; G. D'Angelo, La chiesa del Gesù e la missione del 1649 in Castellammare di Stabia, "Cultura e Territorio», Annali del Distretto Scolastico 38, $\mathrm{n}^{\mathrm{i}}$ 15-16-17, pp. 125-177.

2. Cfr. G. Di Marco, Librai, editori e tipografi a Napoli nel XVII secolo (Parte I), «La Bibliofilia», vol. 112, $\mathrm{n}^{\circ}$ 1, 2010, pp. 21-61; Id., Librai, editori e tipografi a Napoli nel XVII secolo (Parte II), «La Bibliofilia», vol. 112, $\mathrm{n}^{\circ}$ 2, 2010, pp. 141-184: 172.

3. B. De Rogatis, Il regno de' goti nella Spagna abbattuto e risorto, ovvero La perdita e racquisto della Spagna occupata da' mori, Napoli, Francesco Savio, 1648. Si trascrive, modernizzando grafia e punteggiatura, dall'esemplare digitalizzato della Biblioteca Nazionale di Napoli (coll. Sala Farn. 56.F35).

4. E. Maggi, Le verità del falsificatore: storia, finzione e scontro di culture in Miguel de Luna, in Oriente e Occidente nel Rinascimento, Atti del XIX Convegno internazionale (Chianciano-Pienza, 16-19 luglio 2007), Firenze, Cesati, 2009, pp. 611-623.

5. Com'è noto, l'espulsione dei moriscos (i mori convertiti al cristianesimo) dalla Spagna, decretata da Filippo III nel 1609, si protrae fino al 1613. All'origine del provvedimento vi erano complessi motivi d'ordine politico-culturale oltre che religioso.

6. Sulla storia editoriale e la diffusione di quest'opera si rimanda all'introduzione all'edizione facsimil, usata per le citazioni, a cura di Bernabé Pons: M. De Luna, Historia verdadera del rey don Rodrigo, estudio preliminar L. F. Bernabé Pons, Granada, Universidad de Granada, 2001.

7. B. De Rogatis, Il regno de' goti..., cit., f. a3v.

8. B. De Rogatis, Il regno de' goti..., cit., f. a3v.

9. Sulla tassonomia ed i generi in voga presso i gesuiti, si rimanda a P. Chinchilla (dir.), Lexicon de formas discursivas cultivadas por la Compañía de Jesús, Ciudad de México, Universidad Iberoamericana, 2018 (edizione ebook). La storia di De Rogatis viene citata nel capitolo dedicato alla "Historia de...". 
10. M. De Luna, Historia verdadera..., cit., pp. 3-5.

11. B. De Rogatis, Il regno de' goti..., cit., pp. 9-10.

12. C. Sartori, I libretti italiani a stampa dalle origini al 1800. Catalogo analitico con 16 indici, Cuneo, Bertola e Locatelli Editore, 1990-1994, 7 voll. Consultabile all'indirizzo <www.examenapium.it/ libri/sartori.htm>; il Corago. Repertorio e archivio dei libretti del melodramma italiano dal 1600 al 1900 è consultabile all'indirizzo <http://corago.unibo.it/>, mentre il secondo database, al seguente: $<$ www.librettodopera.it/public/>.

13. Purtroppo non è stato possibile consultarlo. Cfr. G. Mazzocchi, El teatro español en Lombardía a fines del siglo XVII, «Diálogos hispánicos de Amsterdam», nº 8, fasc. 3, 1989, pp. 691-714.

14. A. Sabbatini di Salerno, La Eliata overo L'inganno fortunato, Napoli, Giovanni Vernuccio e Nicola Layno, 1693, f. A6r.

15. I. M. Mancini, Li due martiri mori, Napoli, Monaco, 1720, f. A2v.

16. A. F. Ivaldi, "Sancio", overo gli equivoci del sembiante, in Spagna e dintorni, a cura di Maria Grazia Profeti, Firenze, Alinea, 2000, pp. 265-304. Cfr. anche <http://corago.unibo.it/opera/ APC0003687>.

17. Il Sancio, Genova, s. n., 1671, ff. A4v-A7v.

18. G. B. Bottalino, Il Roderico, Milano, Federico Maietta, 1685 ( $2^{\mathrm{a}}$ ed.), pp. 5-7.

19. L. Allacci, Drammaturgia di Lione Allacci accresciuta e continuata fino all'anno MDCCLV, Venezia, Giambattista Pasquali, 1755, p.675. L'argomento storico è registrato parzialmente anche da S. Franchi, Drammaturgia romana, Roma, Edizioni Storia e Letteratura, 1988-1997, 2 voll., vol. II, pp. 512-513. Cfr. anche la tesi di dottorato: L. Ambrosio, Drammi, commedie e favole musicali all'ombra del Colosseo. Modi e forme dell'opera a Roma tra il 1668 e 1689, Pavia, Università di Pavia, 2017, p. 40.

20. G. Malatesta Garuffi (riminese), Il Rodrigo, Dramma per musica d'un solo personaggio, Roma, Tinassi, 1677, pp. 8-11.

21. E. Selfridge-Field, A New Chronology of Venetian Opera and Related Genres, Stanford (California), Stanford University Press, 2007, pp. 236-237; P. Fabbri, Il secolo cantante. Per una storia del libretto d'opera in Italia nel Seicento, Roma, Bulzoni, 2003, p. 298.

22. D. Cupeda, L'amar per virtù, Vienna, Susanna Cristina vedova di Matteo Cosmerovio, 1697, ff. A3r-A4r

23. Egilina, Milano, Giovan Battista Bianchi, 1793, f. [A2r] o p. [5].

24. Per fare un solo esempio, il nome di Cipriotti si affianca in molti paratesti al più noto Francesco Silvani (si veda L'Innocenza difesa) ed è pure il dedicante dell'edizione lodigiana de La fede ne' tradimenti del 1697. Entrambi i libretti mettono in scena nuclei storici spagnoli, esclusi da questo saggio, che derivano da De Rogatis e vedono protagonista l'altra Anagilda.

25. P. Fabbri, Il secolo cantante, cit., p. 299.

26. Per la diffusione di alcuni miti ispanici trattati da De Rogatis, si rimanda L. Romero Tobar, Temas literarios hispánicos, Zaragoza, Universidad, 2013-2015, 2 voll.

\section{RIASSUNTI}

Il saggio avvia una ricerca sull'opera del padre gesuita Bartolomeo De Rogatis intitolata Il regno de' goti nella Spagna abbattuto e risorto, ovvero La perdita e racquisto della Spagna occupata da' Mori diffusa in Italia a partire dal 1648. In essa si trasmette, partendo anche da fonti spagnole 
(in particolare, l'opera di Miguel De Luna), una visione di miti identitari e culturali ispanici. Il filtro di lettura di De Rogatis è successivamente rintracciabile negli argomenti dei libretti del Sei e Settecento, di cui si traccia il corpus relativo alle vicende di Rodrigo, ultimo re dei goti.

L'étude se veut une recherche sur l'œuvre du père jésuite Bartolomeo De Rogatis intitulée Il regno de' goti nella Spagna abbattuto e risorto, ovvero La perdita e racquisto della Spagna occupata da' Mori, qui circule en Italie à partir de 1648. Par cette œuvre on transmet, même en puisant dans des sources espagnoles (en particulier l'œuvre de Miguel De Luna), une vision de l'identité et des mythes culturels hispaniques. Le philtre de lecture de De Rogatis se retrouvera ensuite dans les sujets des livrets d'opéras des $\mathrm{XVII}^{\mathrm{e}}$ et $\mathrm{XVIII}{ }^{\mathrm{e}}$ siècles, où on définit le corpus relatif aux événements de Rodrigo, dernier roi des Goths.

This paper begins a research on the work of the Jesuit Bartolomeo De Rogatis entitled Il regno de' goti nella Spagna abbattuto e risorto, ovvero La perdita e racquisto della Spagna occupata da' Mori spread in Italy since 1648. In it is conveyed, also from Spanish sources (in particular, the work of Miguel De Luna), a vision of Hispanic identity and cultural myths. The reading filter of De Rogatis can be subsequently found in the subjects of the booklets of the sixteenth and eighteenth centuries, of which we trace the corpus relative to the events of Rodrigo, the last King of the Goths.

\section{INDICE}

Mots-clés : Bartolomeo De Rogatis, Miguel De Luna, histoire d'Espagne, mélodrames italiens, Rodrigo, dernier roi des Goths

Parole chiave : Bartolomeo De Rogatis, Miguel De Luna, Storia della Spagna, Melodrammi italiani, Rodrigo, re goto

Keywords : Bartolomeo De Rogatis, Miguel De Luna, Spanish History, Italian Librettos, Rodrigo, last King of the Goths

\section{AUTORE}

\section{ELENA E. MARCELLO}

Università degli studi Roma Tre

elena.marcello@uniroma3.it 